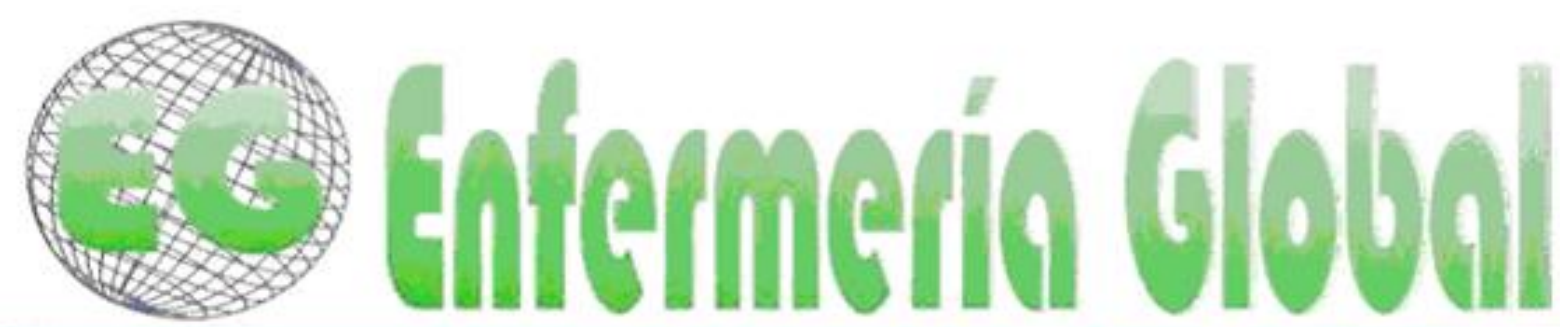

\title{
Percepción de la actividad física en estudiantes de enfermería frente a fisioterapia
}

Perception of physical activity among nursing students versus physiotherapy

\section{*Moreno Collazos, Jorge Enrique ${ }^{* *}$ Cruz Bermúdez, Harold Fabián}

\author{
*Fisioterapeuta, Especialista en Rehabilitación Cardiopulmonar, Magister en Ciencias de la Actividad \\ Física y Deporte, Docente de la Facultad de Enfermería y Rehabilitación, Universidad de La Sabana, \\ Chía-Colombia. E-mail: jorge.moreno2@unisabana.edu.co ${ }^{* *}$ Enfermero, Especialista en Estadística \\ aplicada. Doctor en Ciencias de la Salud. Jefe de Epidemiologia. Instituto del Corazón de \\ Bucaramanga. Colombia.
}

Palabras clave: Estudiantes; ciencias de la salud; motivación (Fuente: DeCs - Bireme) Keywords: Students; health sciences; motivation

\section{RESUMEN}

La actividad física en la actualidad se convierte en una estrategia de prevención de enfermedades, la percepción de los profesionales en formación se convierte en un proceso importante al momento de generar estrategias de intervención en la población.

Objetivo: Establecer la percepción frente a la realización de actividad física en estudiantes de dos programas de ciencias de la salud.

Método: Se realizó un estudio de corte transversal descriptivo e inferencial en estudiantes de primero y segundo semestre de la Universidad de la Sabana. Chía, Colombia.

Resultados: La población de estudio estuvo conformada por 99 estudiantes; en la evaluación de la percepción frente a la actividad física se evidencian diferencias estadísticamente significativas entre los estudiantes $(p=0,000)$

Discusión: La percepción frente a la actividad física es importante al momento de generar estilos de vida saludable en la población, la actividad física es un factor determinante en estilos de vida saludable.

\section{ABSTRACT}

Physical activity now becomes a disease prevention strategy, the perception of undergraduate students becomes an important process when generating intervention strategies in the population. 
Objective: To establish the perception towards the realization of physical activity for students in two programs in health sciences.

Method: A descriptive and inferential study was performed in cross section from first and second half of the Universidad de la Sabana. Chia, Colombia.

Results: The study population consisted of 99 students; the evaluation of perception from physical activity statistically significant differences between students $(p=0.000)$

Discussion: Perception from physical activity are important when generating healthy lifestyles in the population, physical activity is a key factor in healthy lifestyles.

\section{INTRODUCCIÓN}

La actividad física es en la actualidad un factor importante, el momento de generar estilos de vida saludable en la población, se reconoce la práctica de la actividad física como un factor fundamental dentro de los procesos de promoción y prevención que debe implementar los profesionales de la salud, adicionalmente se concibe como un estilo de vida saludable ${ }^{1}$.

La práctica de la actividad física se encuentra relacionada con las motivaciones y percepciones de la población acerca de la misma, en los profesionales de salud en formación es indispensable indagar sus acercamientos, percepciones y mitos frente a su práctica, ya que es importante concebirse como una práctica regular que se realice en intensidades de moderadas a vigorosas, durante el tiempo libre o de ocio y en las actividades de la vida diaria ${ }^{2}$. La realización de la actividad física debe implementarse tanto en el hogar como en el trabajo y en el caso de los estudiantes en formación se deberán generar espacios durante la formación académica con el fin de buscar beneficios para la salud en general, permite experimentar emociones placenteras y sumada a una dieta sana y una actitud positiva ante la vida, en un medio ambiente que fomente estilos de vida saludables, contribuye a mejorar la calidad de vida de la población $n^{3,4}$.

El estudio de las percepciones frente a la actividad física es un tema de gran interés en la actualidad, los comportamientos relacionados con salud y la influencia que pueden tener en factores personales, del entorno próximo y sociocultural al que pertenecen los individuos son indispensables al momento en que el profesional realice educación en salud encaminada a modificar estilos de vida en la población. Los profesionales de enfermería y fisioterapia se convierten en actores importantes y articulados al momento de proponer programas en donde el principal objetivo será implementar la actividad física en la población como un factor protector ${ }^{5}$. Es un reto de las instituciones de educación superior generar espacios en donde los estudiantes practiquen actividad física con el fin de dar a conocer por medio de un aprendizaje vivencial los beneficios que esta trae para la salud; el éxito también de los programas será influenciado por percepciones, las cuales se convierten en procesos mediante los cuales se registra todo con lo que se está interactuando, dando importancia también en los estudiantes a los valores, las tradiciones, las vivencias y los conocimientos, que tienen los individuos sobre determinados aspectos de la vida relacionados con la práctica de actividad física ${ }^{6.10}$.

Por lo anterior el objetivo de este estudio fue establecer la percepción frente a la realización de actividad física en estudiantes de dos programas de ciencias de la salud. 


\section{MATERIALES Y MÉTODOS}

Se realizó un estudio de corte transversal descriptivo e inferencial en estudiantes de primero y segundo semestre de la Universidad de la Sabana. Chía, Colombia, en periodo de abril de 2013 dentro del programa de Respira Sabana organizado por la Facultad de Enfermería y Rehabilitación. La muestra estuvo conformada por estudiantes activos del programa de enfermería y fisioterapia de la universidad, adicionalmente aquellos que aceptaron participar en la encuesta.

Para la obtención de la información se construyó una encuesta para auto diligenciamiento que buscaba evaluar la percepción de los participantes a la actividad física. Con el fin de validar los datos y evitar sesgos se realizó una prueba piloto con 20 sujetos donde se realizó el ejercicio de sensibilización al tipo de pregunta con el fin de realizar los ajustes pertinentes.

Como proceso previo a la aplicación de la encuesta final se sometió el instrumento a un proceso de validación facial y de constructo con el fin de obtener una mayor calidad en la información, para lo cual se tuvo en cuenta la evaluación de 10 expertos en el área de actividad física, todos con nivel mínimo de formación de maestría y experiencia en el área de interés quienes calificaron las preguntas con los siguientes valores de pertinencia: $1=$ no pertinente, $2=$ poco pertinente, $3=$ pertinente y $4=$ muy pertinente. Para la operacionalización de los datos y dar validez al proceso se utilizaron las recomendaciones de Lawshe 2005, quien establece que el valor mínimo para la validación es de 10 expertos y realizar la validación de contenido de un instrumento se debe obtener como mínimo una puntuación de 0,62. Para el cálculo se utilizó la fórmula: Índice de Validez de Contenido IVC= Numero de ítems con puntuación entre 3 y 4 / Número total de Ítems. Esta fórmula se aplicó para cada calificación dada por los expertos y luego se aplicó la formula; Índice de validez de contenido general IVCG = Suma del IVC calculado para cada experto / número de expertos. Después de la evaluación se obtuvo un puntaje de 0,87 por lo cual se decidió aplicar el instrumento ${ }^{11}$.

Las variables del estudio fueron; Edad [años], peso [kg], talla [metros], género, programa académico, estrato socioeconómico, semestre, Îndice de masa corporal [IMC formula: peso/talla*talla], las tras fueron las percepciones evaluadas frente a la actividad física definidas cono variables dicotómicas de [SI - NO]

\section{Análisis estadístico}

En el análisis descriptivo se aplicaron medidas de tendencia central y dispersión para variables cuantitativas y frecuencias absolutas y relativas para variables cualitativas, el análisis se realizó en todos los casos bajo supuestos de normalidad aplicando test de Shapiro-Wilk, se utilizó una confianza del 95\% durante todo el análisis, para comparar el IMC de cada programa se utilizó la prueba T-Student, en el análisis bivariado se realizó una tabla de contingencia con el estadístico Chi-Cuadrado con el fin de establecer la significancia estadística entre las poblaciones a comparar. En el análisis de los datos se realizó el paquete estadístico SPSS de IBM versión 19,0.

Dentro de las consideraciones éticas todos los sujetos aceptaron participar en el estudio, El desarrollo de la investigación cuenta con el aval de la institución y el comité de ética e investigación, la investigación se enmarca en la categoría de investigación sin riesgo puesto que fue un estudio descriptivo según la resolución №. 
008430 de 1993 del Ministerio de Salud de Colombia que establece las normas científicas, técnicas y administrativas para la investigación en salud.

\section{RESULTADOS}

La población de estudio estuvo conformada por 99 estudiantes de los programas de fisioterapia y enfermería de primero y segundo semestre; el promedio de edad fue $18,03 \pm 1,54$ (IC95 17,72 - 14,34) años, el género predominante es el femenino con un $86 \%(n=85)$, el estrato socioeconómico de mayor presentación es el medio, la mayor proporción de estudiantes es del programa de fisioterapia y el semestre que más participo en la actividad fue el primero. El peso promedio fue 62,70 $\pm 7,16$ (IC95 $61,27-64,13$ ) kilogramos y una talla 1,62 $\pm 0,032$ (IC95 1,61 - 1,63) metros (Tabla 1)

Tabla 1. Estadísticos descriptivos de la población de estudio

\begin{tabular}{clcc}
\hline \multicolumn{2}{c}{ VARIABLES } & $\mathrm{N}$ & $\%$ \\
\hline \multirow{2}{*}{ Género } & Masculino & 14 & $14 \%$ \\
& Femenino & 85 & $86 \%$ \\
\hline \multirow{3}{*}{$\begin{array}{c}\text { Estrato } \\
\text { socioeconómico }\end{array}$} & Medio & 75 & $24 \%$ \\
& Alto & 0 & $0 \%$ \\
\hline Programa & Fisioterapia & 64 & $65 \%$ \\
académico & Enfermería & 35 & $35 \%$ \\
\hline Semestre & 1 & 65 & $66 \%$ \\
& 2 & 34 & $34 \%$ \\
\hline
\end{tabular}

Se encontró que al comprar los IMC por programa académico hay diferencias estadísticamente significativas $(p=0,000)$ entre los participantes del programa de enfermería vs fisioterapia.

TABLA 2. Comparación de IMC por programa académico

\begin{tabular}{lcccccc}
\hline & & & Diferencia & \multicolumn{2}{c}{ IC 95\% } & \multirow{2}{*}{ Valor P } \\
\cline { 6 - 7 } & $t^{*}$ & gl & de medias & Inferior & Superior & \\
\hline IMC Enfermería & 46,499 & 34 & 24,1673299 & 23,111095 & 25,223565 & \multirow{2}{*}{0,000} \\
IMC Fisioterapia & 86,335 & 63 & 23,4694476 & 22,926218 & 24,012677 & \\
\hline
\end{tabular}

${ }^{*}$ Prueba T - Student

En el análisis bivariado, se encontraron diferencias estadísticamente significativas entre los estudiantes del programa de fisioterapia frente a enfermería en la pregunta "Considera importante la actividad física para su salud" $(p=0,000)$ el total de los estudiantes de enfermería contestaron "NO" $(n=35)$, "Tiene en cuenta el aporte energético de los alimentos que consume a diario" $(p=0,000)$ el total de estudiantes del programa de enfermería contestaron "NO" $(n=35)$, En la última semana ha realizado actividad física $(p=0,000)$ tan solo el $7 \%$ de los estudiantes de enfermería habían realizado actividad física, solo el $11 \%$ de los estudiantes de enfermería practican un deporte diferente a fisioterapia donde la distribución es más uniforme, "Considera importante dentro de su formación la práctica de actividad física el $77 \%$ de 
los estudiantes de fisioterapia lo consideran importante $(p=0,000)$, "Usted es promotor de la actividad física en la familia" y "Si existiera un programa de actividad física asistiría" se encuentran diferencias entre las percepción de los dos programas de formación $(p=0,000)$ (Tabla 3).

Tabla 3. Comparación entre programas académicos de variables del estudio

\begin{tabular}{|c|c|c|c|c|c|c|}
\hline \multirow{3}{*}{ VARIABLE } & & \multicolumn{4}{|c|}{ Programa académico } & \multirow{3}{*}{$\begin{array}{l}\text { P. } \\
\text { valor* }\end{array}$} \\
\hline & & \multicolumn{2}{|c|}{ Fisioterapia } & \multicolumn{2}{|c|}{ Enfermería } & \\
\hline & & $\mathbf{n}$ & $\%$ & $\mathbf{n}$ & $\%$ & \\
\hline \multirow[t]{2}{*}{ Género } & Masculino & 8 & $57 \%$ & 6 & $43 \%$ & \\
\hline & Femenino & 56 & $66 \%$ & 29 & $34 \%$ & 0,526 \\
\hline \multirow[t]{2}{*}{ Realiza actividad Física } & $\mathrm{NO}$ & 22 & $40 \%$ & 33 & $60 \%$ & \\
\hline & SI & 42 & $95 \%$ & 2 & $5 \%$ & $0,085^{\star \star}$ \\
\hline \multirow{2}{*}{$\begin{array}{l}\text { Considera importante la actividad } \\
\text { física para su salud }\end{array}$} & $\mathrm{NO}$ & 7 & $17 \%$ & 35 & $83 \%$ & \\
\hline & $\mathrm{SI}$ & 57 & $100 \%$ & 0 & $0 \%$ & 0,000 \\
\hline \multirow{2}{*}{$\begin{array}{l}\text { Tiene en cuenta el aporte } \\
\text { energético de los alimentos que } \\
\text { consume a diario }\end{array}$} & NO & 12 & $26 \%$ & 35 & $74 \%$ & \\
\hline & SI & 52 & $100 \%$ & 0 & $0 \%$ & 0,000 \\
\hline \multirow[t]{2}{*}{ Clasificación de IMC } & Normopeso & 34 & $53 \%$ & 14 & $40 \%$ & \\
\hline & Sobrepeso & 30 & $47 \%$ & 21 & $60 \%$ & 0,212 \\
\hline \multirow{2}{*}{$\begin{array}{l}\text { Usted considera que se encuentra } \\
\text { en sobrepeso }\end{array}$} & $\mathrm{NO}$ & 16 & $67 \%$ & 8 & $33 \%$ & \\
\hline & $\mathrm{SI}$ & 48 & $64 \%$ & 27 & $36 \%$ & 0,812 \\
\hline \multirow[t]{2}{*}{ Consume cigarrillo } & $\mathrm{NO}$ & 35 & $57 \%$ & 26 & $43 \%$ & \\
\hline & $\mathrm{SI}$ & 29 & $76 \%$ & 9 & $24 \%$ & 0,055 \\
\hline \multirow{2}{*}{$\begin{array}{l}\text { En la última semana ha realizado } \\
\text { actividad física }\end{array}$} & $\mathrm{NO}$ & 16 & $36 \%$ & 28 & $64 \%$ & \\
\hline & SI & 48 & $87 \%$ & 7 & $13 \%$ & 0,000 \\
\hline \multirow[t]{2}{*}{ Practica algún deporte } & $\mathrm{NO}$ & 24 & $44 \%$ & 30 & $56 \%$ & \\
\hline & SI & 40 & $89 \%$ & 5 & $11 \%$ & 0,000 \\
\hline \multirow{2}{*}{$\begin{array}{l}\text { Considera importante dentro de su } \\
\text { formación la práctica de actividad } \\
\text { física }\end{array}$} & $\mathrm{NO}$ & 7 & $28 \%$ & 18 & $72 \%$ & \\
\hline & SI & 57 & $77 \%$ & 17 & $23 \%$ & 0,000 \\
\hline \multirow{2}{*}{$\begin{array}{l}\text { Usted es promotor de la actividad } \\
\text { física en la familia }\end{array}$} & $\mathrm{NO}$ & 10 & $26 \%$ & 29 & $74 \%$ & \\
\hline & SI & 54 & $90 \%$ & 6 & $10 \%$ & 0,000 \\
\hline \multirow{2}{*}{$\begin{array}{l}\text { Si existiera un programa de } \\
\text { actividad física asistiría }\end{array}$} & $\mathrm{NO}$ & 0 & $0 \%$ & 26 & $100 \%$ & \\
\hline & SI & 64 & $88 \%$ & 9 & $12 \%$ & 0,000 \\
\hline
\end{tabular}

\section{DISCUSIÓN}

Se permitieron identificar percepciones importantes en los estudiantes de fisioterapia y enfermería frente a la práctica de actividad física, es indispensable que estas motivaciones iniciales sean intervenidas ya que los profesionales de la salud deben reflejar un estilo de vida saludable al momento de abordar un paciente para hablar sobre estilos de vida saludable ${ }^{12,13}$.

Es claro que las concepciones se van transformado a medida que se dan hechos o cambios que las activan, es indispensable que aunque el IMC es una característica física que puede orientar frente a estilos de vida saludable relacionados con hábitos, estos deben comportarse dentro de parámetros habituales que indiquen las 
actividades realizadas por el profesional de la salud orientadas a cuidar su propia salud. En el caso de la realización de actividad física es importante que el programa de enfermería se apoye en fisioterapia generando estrategias para que los futuros egresados de enfermería consideren importante dentro de su práctica profesional la inclusión de actividad física como un estilo de vida saludable, adicionalmente que los procesos de promoción y prevención se enfoquen adicionalmente ene este factor protector que se debe trabajar en la población.

Aunque la prescripción del ejercicio debe ser dada por profesionales formados en la disciplina, la profesión de enfermería debe incluir estrategias orientadas a que la población acuda a programas de práctica de actividad física, enmarcado dentro de procesos de autocuidado de la salud y mantenimiento de estrategias de mitigación y prevención de la enfermedad, en general la vida saludable ${ }^{14}$.

Al evaluar la percepción en los estudiantes se evidencia una notable asociación que hacen entre la salud y sus determinantes, es importante así mismo que los estudiantes comprendan los factores relacionados con la biología o herencia, el estilo de vida, el entorno social y los servicios de salud, los cuales en países como Colombia se orientan en aspectos de promoción de la salud y prevención de la enfermedad destacando desde la perspectiva personal su compromiso con la salud, con la misma formación académica, la autoestima y los estilos de vida asociados con el cuidado del cuerpo, sin embargo, todas estas aceraciones a la percepción de la actividad física se convierten en acciones prácticas de las cuales dan cuenta y desde donde podrían potenciar su salud y la formación; los programas de enfermería y fisioterapia son un factor determinante de interdisciplinariedad al momento de generar abordaje en factores determinantes de la salud de las personas ${ }^{15-18}$.

No podemos trabajar en los estudiantes cuando entonces existe un doble mensaje, entre lo que pretenden ofrecer a la población los profesionales de la salud y lo que ellos mismo interiorizan en su cuidado de la salud, entonces es cuando hablamos desde enfermería de autocuidado en los pacientes y al evaluar condiciones de cuidado de la salud en nuestros estudiantes en formación la práctica de actividad física no es un factor conducente a la realización de prácticas de cuidado de la salud, podemos decir que hay una contradicción entre el discurso y el de la acción; esto es, el primero no se consolida en la práctica ${ }^{2,5,19}$.

La práctica de actividad física se entrelazan con algunos principios de integralidad, desde lo emocional y actitudinal, pero se sigue considerando en la actualidad como un aspecto de importante trabajo al momento de realizar prácticas saludables, es más la Organización Mundial de la Salud continúa impartiendo esta como una de las medidas más costo efectivas para la prevención de enfermedades cardiovasculares en la población, prevención de sedentarismo y como factor protector para un sin número de patologías que afectan a la población de la actualidad, la realización de actos cotidianos y espontáneos de la vida diaria que implican un gasto energético por encima del basal se deben considerar ya como practicas encaminadas a proteger y mejorar la calidad de vida de las personas ${ }^{1-2}$.

Actualmente existen afirmaciones que buscan explicar porqué algunas personas se adhieren a la práctica de actividad física y otras no, una de estas están relacionadas con personas más activas lo cual ha llevado a establecer las determinantes de la práctica de la AF: por ejemplo factores intrapersonales evaluados desde una aproximación cualitativa en este estudio, sociales y del ambiente físico, en donde aunque los estudiantes de fisioterapia su razón de estudio es el movimiento corporal 
humano, para enfermería es el cuidado de la salud de la población, dos puntos que se encuentran entrelazados fuertemente y que se orientarían a que la percepción frente a la práctica de ejercicio debería tener un comportamiento similar ${ }^{7-10}$.

Desde la investigación integrativa relacionada con la percepción de la actividad física no sólo se ubican problemáticas, sino que permite buscar alternativas para dar respuestas a las mismas en forma contextualizada y pertinente, es necesario que se trabaje desde la academia en la apropiación del fundamento teórico que conforman la actividad física como un practica saludable, y orientar los programas hacia la necesidad de hacer visible en la comunidad universitaria la promoción de la salud, prevención de la enfermedad y calidad de vida ${ }^{20-21}$.

\section{CONCLUSIONES}

En general, la población de estudio hace referencia a la actividad física, se encuentran diferencias entre la población que ingresa al programa de fisioterapia y enfermería frente a la percepción de la actividad física dentro de sus estilos de vida saludable. Aunque las dos carreras pertenecen a las ciencias de la salud, es indispensable que los profesionales de enfermería identifiquen la actividad física como una práctica importante dentro de los procesos de promoción de la salud y prevención de la enfermedad, así como que dentro de procesos de intervención orientados al autocuidado la práctica de la actividad física se introduzca dentro de su propio estilo de vida y que esta sea incluida con mayor fuerza dentro del proceso de formación académica.

Es indispensable que los futuros profesionales de la salud al momento de generar estrategias de mitigación frente al sedentarismo o la percepción de la actividad física, estos cuenten con una idea clara y una convicción segura frente a la misma, adicionalmente que su imagen y empoderamiento sea vista por los sujetos de intervención.

Es indispensable trabajar de una manera interdisciplinaria en donde las dos profesiones sean un complemento y se generen intervenciones efectivas dadas por las contribuciones disciplinares que cada una puede aportar.

\section{REFERENCIAS BIBLIOGRÁFICAS}

1. Organización Mundial de la Salud. Estrategia mundial sobre el régimen alimentario, actividad física y salud. Ginebra: OMS, 2002.

2. Organización Panamericana de la Salud. Agenda de salud para las Américas 2008 2017. Washington: OPS/OMS, 2007.

3. Global Advocacy Council of Physical Activity, International Society for Physical Activity and Health. The Toronto charter for physical activity: A global call for action. May 8, 2010. [Citado en 2010 febrero 8]. Disponible en: http://www.jcpaph.org.

4. Keating XD, Guan J, Castro J, Bridges DM. A meta-analysis of college students' physical activity behaviors. J. Am. Coll. Health 2005; 54:116-125.

5. Burke SM, Carron AV, Eys MA. Physical activity context: preferences of university students. Psychology of Sport and Exercise. 2006; 7:1-13. 
6. Elizondo-Armendáriz JJ, Guillén F, Aguinaga I. Prevalencia de actividad física y su relación con variables sociodemográficas y estilos de vida en la población de 18 a 65 años de Pamplona. Rev Esp Salud Pública 2005;79: 559-567.

7. Seo D, Nehl E, Agley J, Ma S. Relations between physical activity and behavioral and perceptual correlates among midwestern college students. Journal of American College Health 2007; 56:187-197.

8. Vargas-Zárate M, Becerra-Bulla F, Prieto-Suárez E. Evaluación antropométrica de estudiantes universitarios en Bogotá, Colombia. Rev. salud pública. 2008;10:433-442.

9. Franko DL, Cousineau TM, Motivation self-efficacy, physical activity and nutrition in college students: Randomized controlled trial of an internet based education program. Prev Med 2008; 47:369377.

10. Brunt AR, Rhee YS. Obesity and lifestyle in U.S. college students related to living arrangements. Appetite 2008; 51:615621.

11. Lawshe, C. pruebas psicotécnicas en la selección de personal. Facultad de psicología. Pontificia Universidad javeriana,. 2005 pág. 75.

12. Hernández R, Collado F, Baptista L. Metodología de la investigación. 5a ed. México: McGraw Hill, 2010.

13.Talavera M. El concepto de salud, definición y evolución (Monografía en Internet). [Citado en 2008 agosto 20]. Disponible en:http://www.unal.edu.co/medicina/Departamentos/Pediatria/Pediatria/EL\%20 CONCEPTO\%20DE\%20SALUD.doC

14.López J, Fernández A. Fisiología del ejercicio. Madrid: Editorial Médica Panamericana, 2008.

15.Jiménez A, Montil M. Determinantes de la Práctica de Actividad Física: Bases, Fundamentos y Aplicaciones. Editorial Dykinson. 1르. ed. España 2006.

16. Ministerio de la Protección Social de Colombia. Guía para el desarrollo de programas intersectoriales y comunitarios para la promoción de la actividad física. Bogotá: Ministerio de la Protección Social de Colombia, 2004.

17. Ministerio de la Protección Social de Colombia. Decreto 3039 de 2007. Plan Nacional de Salud Pública 2007-2010. Bogotá; 10 de agosto de 2007.

18. Ciolac EG. Exercise training as a preventive tool for age-related disorders. Clinics 2013; 68: 710-7.

19. Garber CE, Blissmer B, Deschenes MR, Franklin BA, Lamonte MJ, Lee IM, Nieman DC, Swain DP; American College of Sports Medicine position stand. Quantity and quality of exercise for developing and maintaining cardiorespiratory, musculoskeletal, and neuromotor fitness in apparently healthy adults: guidance for prescribing exercise. Med Sci Sports Exerc 2011; 43: 133459.

20. Artinian NT, Fletcher GF, Mozaffarian D, Kris- Etherton P, Van Horn L, Lichtenstein AH, Kumanyika S, Kraus WE, Fleg JL, Redeker NS, Meininger JC, Banks J, Stuart-Shor EM, Fletcher BJ, Miller TD, Hughes S, Braun LT, Kopin LA, Berra K, Hayman LL, Ewing LJ, Ades PA, Durstine JL, Houston-Miller N, Burke LE; American Heart Association Prevention Committee of the Council on Cardiovascular Nursing. Interventions to promote physical activity and dietary lifestyle changes for cardiovascular risk factor reduction in adults. Circulation 2010; 122: 406-41.

21.Brown WJ, Leung J, McCaul KA, Flicker L, Almeida O, PHankey GJ, Lopez D, Dobson AJ Physical activity and all-cause mortality in older women and men. $\mathrm{Br}$ J Sports Med 2012 Jan 4; 46: 664-668. 
Recibido: 21 de octubre 2014; Aceptado: 24 de noviembre 2014 